\title{
Introduction à l'Etude Clinique des Crises Epileptiques Rétrorolandiques
}

\author{
J. Baucaud, J. Talairach, C. Munari, T. Giallonardo et P. Brunet
}

\begin{abstract}
RÉSUMÉ: Nous avons analysé chez 145 malades épileptiques, 800 crises cliniques et électriques (540 spontanées et 260 induites) dont la décharge initiale a mis en jeu les territoires rétrorolandiques. Ces patients ont tous été étudiés suite à la mise en place d'électrodes intracrâniennes réalisée selon la méthode stéréotaxique (SEEG) décrite par Talairach et al., 1974. Les explorations SEEG ont permis d'enregistrer des crises dont l'origine a été centro-pariétale (64 patients), pariétales, ${ }^{49}$ occipitales ${ }^{15}$ et mixtes. ${ }^{15}$ Parmi ces malades, 108 ont subi l'exérèse d'un foyer épileptique (69 à droite, 39 à gauche) avec un taux de guérison de 65\% (suppression des crises ou crises occasionnelles chez 70 patients, recul $>3$ ans). Les principales catégories de manifestations cliniques témoignant de la désorganisation paroxystique de ces territoires sont présentées. Plus particulièrement, et à partir de crises dont la décharge a affecté le cortex supratemporal (portion fronto-pariétale), le rôle du complexe operculo-insulaire dans l'organisation fonctionnelle chez l'homme est discuté. L'étude de l'organisation spatio-temporelle des décharges et de leurs corollaires cliniques affectant les régions corticales rétrorolandiques peut permettre un diagnostic topographique différentiel.
\end{abstract}

\begin{abstract}
An introduction to the clinical study of postrolandic epileptic seizures. We reviewed, in 145 epilectics studied with SEEG, 800 clinical and electrographic seizures originating from the post-rolandic areas (590 spontaneous $\mathrm{Sz}$ and 260 induced by stimulation). The intra-cranial electrodes were implanted using a technique described by Talairach et al. (1974). Seizure onsets were recorded in the centro-parietal region (64 patients) in the partietal 49 and in the occipital region. ${ }^{15}$ Additionally in 15 patients, mixed Sz onset were recorded. One hundred and eight (108) patients underwent surgical removal of their epileptic focus. (69 on the right, 39 on left.) $65 \%$ were cured ( $\mathrm{Sz}$ free or occas $\mathrm{Sz}$, f/up 3 years). The main ictal cal features are discussed. Emphasis is placed on the role of the operculo-insular cortex in the functional organization in man, based on $\mathrm{Sz}$ arising from the supra-temporal (fronto-pariental) cortex. The study of the pattern of onset and spread of seizures originating in the post-rolandic areas and of their clinical correlates allow a topographic differential diagnosis.
\end{abstract}

Can. J. Neurol. Sci. 1991; 18: 566-569

Il n'est pas possible, dans le cadre de cette contribution, de rendre compte d'un probleme aussi vaste et complexe que celui de l'organisation sémiologiques des crises dont la décharge affecte primitivement le cortex des différents territoires postérieurs des hémispheres (régions parietales. Occipitales et temporales postérieures).

Nous allons simplement rappeler, en nous aidant de la littérature, les grandes catégories de troubles rapportables à la désorganisation paroxystique de ces "provinces" par une décharge enregistrée au cours des explorations SEEG pratiquées dans le but de discuter une intervention chirurgicale chez des malades presantant une épilepsie rebelle. Aussi cette schematisation ne peut pas permettre une étude de l'organisation spatio-temporelle des décharges et de leurs corrollaires cliniques. Elles peut seulement orienter un diagnostic topographique vers les régions postérieures des hémispheres. Pour illustrer les implications neurophysiologiques de la méthode que nous avons prèconisée (et surtout pour rehare hommage á l'Ecole du M.N.I.) nous donnerons ensuite quelques indications sur le rôle fonctionnel que semble jouer. Chez l'homme, le "complexe operculo-insulaire" dont on sait que W. Penfield et $\mathrm{H}$. Jasper et leurs collaborateurs ${ }^{1-3}$ lui ont consacre des travaux primordiaux.

\section{Population et Methodes}

\section{Population}

Notre population comporte 145 malades ayant présenté 800 crises (540 spontanées, 210 induites par la stimulation électrique directe et 50 provoguées par la Mégimide) dont la décharge met en jeu initiallement les territoires rétrorolandiques.

Le choix de ces malades à été realisé en nous fondant sur la cohérence des explorations SEEG (nombre, emplacement des electrodes, etc.) et la fiabilite (corrélations correctement établies) des données anatomo-électro-cliniques obtenues lors des accès.

Les caractéristiques générales de cette série ne diffèrent pas de celles de la population générale des malades explorés et opérés. ${ }^{4.5}$ En utilisant une catégorisation anatomique grossière,

From the Institut National de la Santé et de la Recherche Médicale, Unité de Recherches sur l'Epilepsie

Reprint requests to: Dr. J. Baucaud, Institut National de la Santé et de la Recherche Médicale, Unité de Recherches sur l' Epilepsie, Centre Paul Braca, 2 ter, rue d'Alésia, 75014 Paris, France 
on peut considérer que les explorations SEEG ayant permis d'enregistrer des crises ont surtout porté sur les régions suivantes:

- centro-pariétales:

(en particulier, gyrus post-central)

- pariétales:

- occipitales:

- mixtes (jonctions parieto-temporale post: jonctions pariéto-occipitale: jonctions pariétooccipitale; jonctions occipito-temporale post; carrefour temporo-pariéto-occipital:

On doit noter que la jonction pariéto-temporale (au niveau de l'hemisphere dominant), l'aire 17 et le pôle occipital et les portions pariétales parasagittales ont été explorées, pour des raisons de sécurité avec un nombre restreint d'électrodes. Ces investigations ont conduit à 108 exéréses ( 69 à droite, 39 à gauche) avec un taux de quérison de 65\% (suppression des crises ou crises occasionelles chez 70 malades avec un recul supérieur à 3 ans).

\section{Methodes}

La mise en place des électrodes intracraniennes à contacts étagés (environ 10 électrodes par exploration comportant chacune environ 10 plots d'enregistrement et de stimulation) a été réalisée selon la méthode stéréotaxique décrite par l'un de nous et utilisée récemment dans l'interprétation des images du scanner et de I'IRM. ${ }^{6}$

Les explorations SEEG ont été pratiquées selon les principes souvent définis par notre groupe. ${ }^{5}$ Nous devons seulement rappeler que la stratégie d'investigation est, pour nous, déterminée dans chaque cas, par les données obtenues lors des examens préalables et, en particulier, grâce à l'étude des corrélations anatomo-électro-cliniques qui caractérisent les accés enregistrés sur le scalp confrontes à l'existence (ou non) d'accidents pointus intercritiques et à la présence (ou non) de lésions (examens neurologiques et neuroradiologiques).

Il est évident que l'analyses des troubles des "fonctions supérieures" au cours d'une crise de durée breve et dans les conditions d'examen post-critique est difficile et incomplété et exige une autre approche de celle de la Neuropsychologie des affections "chroniques". Pourtant les modalités particuliéres de désorganisation corticale aigue par une décharge et les diverses stratégies de restructuration peuvent nous fournier des indications intéressantes sur certains aspects du fonctionnemént cérébral si on compare les effets respectifs d'une décharge d'origine de propagation et de durée identiques ou différentes chez le même malade et chez tous les autres.

\section{Resultats}

A. Généralitées sur les crises rétrorolandiques

I- Troubles somato-sensitives (engourdissements, fourmillements, etc.)

I-1- Limités à une portion de l'hemicorps contralateral à la décharge : Gyrus post-central

1-2- A "marche jacksonnienne" : Gyrus post-central

I-3- Hémicorporels : Gyrus post-central

I-4- Bilatéraux : Cavité buccale et langue : Aire somatosensitive II

Trois remarques :

a) Dans notre experience, la propagation de la décharge au niveau de la pariétale ascendante se fait rarement, du fait même de l'organisation anatomo-fonctionnelle de Rolando chez l'homme, selon un seul plan qui respecterait la somatotopie classique.

b) La représentation sensitive controlatérale des organes génitaux externes nous parait se faire au niveau de la portion basse du lobule précentral au dessous de celle des orteils.

c) L'organisation fonctionnelle de la region "operculo-insulaire" sera envisagée plus loin.

II- Troubles perceptuels

II-1- Visuels (hallucinations, illusions (métamorphopsies)

Il-1-a-i- Hallucinations simples (région de l'aire 17)

- points lumineux, papillons, etc.

- intensité variable

- souvent colorées (uniformes ou non)

- contralatérales

- localisées dans le champ visuel

- mouvantes (rarement fixes)

- quadranopsie, hemianopsie (per et post-ictal)

II-1-a-ii- Hallucinations complexes (régions aires 18 et 19) déplacement latéral

- parfois scénes mais sans rêve

- parfois hemianopsique

II-1-b- Illusions (metamorphopsies dans un sens littéral)

II-1-b-i- Simples

- taille : jonction occipito-pariétale ou pariétale postérieur (nette prédominance droite)

- Ondulation : idem

- modification des couleurs : idem : prédominance

- mouvement : idem (nette prédominance droite)

- vision renversée : cortex occipital

- fragmentation des contours :

II-1-b-ii- Complexes

- déformation des images : jonction occipito-pariétale (droite)

- teleopsie et polyopie : cortex occipital

Remarque : La distinction entre illusions simples et complexes, en grande partie arbitraire, souléve des problèmes complexes.

II-2- Gustatifs

Les hallucinations et les illusions gustatives sont en rapport avec une désorganisation de la région operculo-insulaire dans sa portion postérieure (pariétale)

II-3- Vertigineux

Il faut, semble-t-il, différencier les manifestations vertigineuses "vraies" (sensations de déplacement ou de rotation du monde extérieur dans une direction données) des "pseudo-vertiges" (sensation de malaise avec impression de déplacement du corps sans systématisation, "ça tourne").

Dans le premier cas on à affaire à une désorganisation de la région pariétale inférieure externe et (ou) interne, alors que dans le second, la participation des aires 41-42 (Tl) paraît indespensable.

\section{II-4- "Auditifs"}

S'il est clair que la perturbation des gyri de Heschl et du planum temporale joue un rôle essentiel dans la survenue des phénomènes hallucinatoires auditifs, on comprend qu'une décharge qui affecte initiallement le cortex pariétal postéroinférieur puisse $s$ accompagner de manifestations du même type. 
Seuls les signes d'accompagnement (voisinage et propagation) peuvent permettre un diagnostic différentiel éventuel.

\section{III- Troubles gnosiques}

On peut distinguer de façon très schématique les troubles paroxystiques de l'image de sol et du monde extérieur par :

- transformation corporelle

- illusion de déplacement

- membre fantôme

- illusions heautoscopique

- agnosie spatiale unilatérale (per et post-critique)

Ces manifestations nous sembient en rapport avec une désorganisation du lobe pariétal droit ("mineur") et admettre, comme l'a suggéré Hecaen, une prévalence somatotopique.

L'existence d'un syndrome de Gerstmann paroxystique (région pariétale gauche) reste discutée.

IV- Troubles douloureux somatiques latéralisés

On doit, semble-t-il, distinguer deux modalités de phénoménes douloureux paroxystiques.

1) Sensations douloureuses limitées (controlatérales à la décharge)

- Désorganisation primaire de la région du gyrus post-central

- Substitution ou association à des paresthésies diverses de siège identique

- Organisation somatotopique

2) Phénomènes douloureux de type "somatognosique"

Dans ce cas la douleur serait un équivalent somatognosique (selon Hecaen et Angelergues) et admet une très nette prévalence pariétale droite de la décharge.

V- Dreamy-state à composante visuelle prévalente

On doit, à notre avis, distinguer les hallucinations et (ou) les illusions visuelles complexes sans recollection paroxystique (qui impliquent une désorganisation du cortex occipital ou de la jonction occipito-temporale) des recollections à composante visuelle prédominante.

On sait, que pour W. Penfield, ces manifestations sont en rapport avec une perturbation du néo-cortex alors que pour $P$. Gloor elles sont surtout sous la dependance des structures temporales internes et, en particulier, du noyau amygdalien.

Nos données sont plutôt en faveur d'une perturbation simultanée du cortex occipital ou occipito-temporal, de la corne d'Ammon et du noyau amygdalien.

En schématisant on pourrait dire qu un dreamy-state à composante visuelle est la résultante d'une difficulté d'intégration mémorielle (corne d'Ammon) de certaines composantes visuelles (cortex occipito-temporal) dans un contexte affectif particulier (noyau amygdalien).

VI- Manifestations versives et posturales

VI-1- Phénomènes versifs

VI-1-a- Crises oculo-cloniques, toniques ou tonicocloniques (controlatérales)

- région péri et parastriée

- propagation au système optique "secondaire"

- entrainement fréquent de la tête

VI-1-b- Adversions conjuguées de la tête et des yeux (prédominance homolatérales)

- région du carrefour temporo-pariéto-occipital

VI-2- Phénomènes posturaux

VI-2-a- Gyration du corps sur l'axe (le plus souvent controlatérale)
Cette manifestation qui est peut être initiallement en rapport avec une désorganisation du contrôle vestibulaire cortical (equivalent vertigineux) parait secondaire à une mise en jeu critique du lobe pariétal dans sa portion inférieure.

VII- Troubles du langage

VII-1- Aphémie ("littérale")

On sait que l'impossibilité pour le malade, au cours d'une crise, de communiquer oralement peut aller de pair avec des décharges affectant des territoires corticaux trés éloignés (régions pariéto-rolandique basse ou régions pariéto-temporale postérieure gauche)

VII-b- Troubles de la compréhension verbale

Désorganisation de la région pariétale basse et temporale postérieure gauche

VII-c- Aphasie de conduction

Prévalence de la désorganisation operculo-insulaire postérieure.

VII-d- Syndrome de disconnexion ("Alexie pure")

Région des carrefours temporo-pariéto-occipital (prévalence occipitale) ; rôle majeur de la propagation homologue controlatérale de la décharge.

\section{B-Organisation fonctionnelle de la région operculo-insulaire}

L'étude systématique de 75 crises dont la décharge affecte le cortex supratemporal de la région operculo-insulaire chez 28 malades dans sa portion fronto-pariétale nous permet de formuler les hypothèses suivantes sur le rôle que jouerait ce "complexe" dans l'organisation fonctionnelle chez l'homme.

I- Intégration gustative (versant postérieur)

II- Modulations corticales des fonctions végétatives sensorimotrices

1) Tractus digestif

a- sensations viscerales (portion postérieure)

- anale

- abdominale

- gastrique

- oesophagienne

- pharyngée

b- activités motrices (portion antérieure)

- salivation

- déglutition

- mastication

c- système respiratoire

- fréquence cardiaque

- sensations précordiales, palpitations

- pression artérielle

- vaso-dilatation et vaso-constriction (face-thoraxtête)

d- thermorégulation

- froid et chaud

e- musculature oculaire intrinsèque

- mydriase et myosis

f- tractus uro-génital;

- sensations génitales (bilatérales)

- sensations génésiques

- erection

- orgasme

- comportement moteur sexuel complexe

- envie d'uriner ou urination 
La définition de l'origine et de la propagation des décharges critiques impliquant cette région permet de soulever de nombreux problèmes anatomo-fonctionnels (et épileptologiques) dont nous ne pouvons évoquer que quelques aspects :

- Les régions corticales rétro et pré-rolandiques de l'opercule central sont étroitement interconnectées (par exemple versants sensitifs et moteurs de la modulation corticale du tractus digestif)

- Les sensations génitales bilatérales (dont les rapports avec l'intégration sensitive des organes génitaux externes au niveau du lobule paracentral nous sont inconnues) nous paraissent relayées par l'effecteur essentiel que constitute le noyau amygdalien sans doute vers les regions hypothalamiques postérieures.

- Le besoin d'uriner ou même l'urination, lors d'un accés partiel d'origine insulaire, est sous le contrôle de la région orbitaire frontale qui elle même projette vers les structures sous corticales, etc.

\section{ConClusion}

Le catalogue que nous avons rappelé des principales catégories de manifestations cliniques témoignant de la désorganisa- tion paroxystique des régions corticales rétrorolandiques ne saurait, en aucun cas, remplacer une étude sémiologique systématique des différents types de crises qui affectent ces territoires.

Il permet simplement de rappeler que la très grande diversité des troubles qui les caractérisent permettent de s'orienter assez bien, en les combinant, dans le diagnostic topographique différentiel.

\section{REFERENCES}

1. Penfield W, Jasper HH. Epilepsy and the Functional Anatomy of the Human Brain. Boston, Little Brown; 1954.

2. Penfield W, Rasmussen T. The Cerebral Cortex of Man. New York, MacMillian; 1950.

3. Penfield W, Faulk ME. The Insula. Brain 1955; 78: 445-470.

4. Talairach J, Bancaud J, Szikla et al. C (1974): Approche nouvelle de la neurochirurgie de 1 épilepsie. Neurochirurgie, 1974. Paris 20 (suppl. 1): 1-240.

5. Bonis A. Long-term results of cortical excision based on stereotactic investigation in severe, drug-resistant epilepsies. Acta Neurochir, 1980; (Wien) 20 (suppl): 55-66.

6. Talairach J, Toumoux P. Co-planar stereotaxic Atlas of the human brain. 3-Dimensional Proportional System. An Approach to Cerebral Imaging. Stuttgart-New York, George Thieme; 1988. 